\title{
Persistent Collections with Customizable EquiVALENCE AND IDENTITY SEMANTICS
}

\author{
Konrad Grzanek \\ IT Institute, University of Social Sciences, Łódź, Poland \\ kgrzanek@spoleczna.pl,kongra@gmail.com
}

\begin{abstract}
Providing a comprehensive set of mechanisms solving the problem of controlling equivalence and identity requires implementing the functionality for non-sequential containers instrumented with the enriched semantics. Functional programming languages, like Clojure, typically miss the functionality by default. The article presents the design considerations, concepts and implementation details of generalized sets and maps aware of the customizable equivalence and identity together with some usage examples.
\end{abstract}

Key words: Equivalence testing, semantics, identity, functional programming, Clojure, persistent collections

\section{Introduction}

Testing object for their equivalence as well as creating criteria of establishing their identity is one of the most important tasks to be realized during mature software implementation process. A developer must consider many factors here, like the typing system of the programming language, either static or dynamic, based on the structural equivalence or using tags. The number and the extent of decisions to be made when designing the identity and equivalence related algorithms is so large and wide that the attempts to make a kind of their uniformization in a single library or API requires special approaches. Namely, the mechanisms must be put in a formal shape consisting of a set of extendable equivalence and identity operators. A lack of these mechanisms even in as mature programming languages as Java and Clojure led to a work on their detailed design and implementation in the latter. 
The results of the undertaking were presented in a paper titled Equivalence in Java and Clojure, Design and Implementation Considerations [1]. Most of it's content focused on:

- the equivalence and identity abstractions,

- implementation details for Java primitive types [3],

- implementation details for java.lang.Number [2] derivatives,

- implementation details for some Clojure [4], [5] reference types (clojure.lang.Ratio, clojure.lang.BigInt),

- implementation details for selected sequential values: instances of java.lang.String, clojure.lang.ISeq, java.util.List, kongra.core.Pair.

The mentioned paper lacked a presentation of concepts as well as implementation elements for other kinds of collections, namely sets and maps (associative containers). The present article may be treated thereafter as a natural continuation of [1] with an aim to present customizable, extendable equivalence and identity realization in the two kinds of containers. One final mark to be made here is we will focus on Clojure persistent sets and maps, abandoning completely other realizations of java.util.Set and java.util.Map interfaces [2].

\section{Equivalence, Identity and the Persistent Collections in Clojure}

Traditionally the functional programming style is associated with a lack of explicitly changeable state. Objects posses value semantics, they are immutable values like the notions in mathematics. This property opens ways to discuss formally the properties of programs and even sometimes makes the properties of programs provable. From the engineer's point of view the lack of state makes achieving programs' correctness easier (in more complex cases possible).

Immutable collections are the core of Clojure data structures [5]. They are called persistent there, with the term persistent meaning their persistence across the operators working on them. In other words, the collection operators in the language are non-destructive, they do not modify their arguments. Another property of the persistent collections is their structure-sharing. This is a pretty old concept, sequences with structure sharing were common in various dialects of Lisp (see [7], [8]). Nowadays data structures other than the sequential ones are known to have the property, e.g. hash-tries [6]. Clojure has the following kinds of persistent collections: 
- sequences, including vectors1

- sets

- maps

- records - tagged objects with a map semantics

As stated earlier, our previous paper [1] presented a realization of customizable equivalence and identity operators for sequences (clojure.lang.ISeq derivatives) and lists including vectors (clojure.lang.PersistentVector $<$ : clojure.lang.APersistentVector $<:$ java.util.List). As a consequence of having these mechanisms:

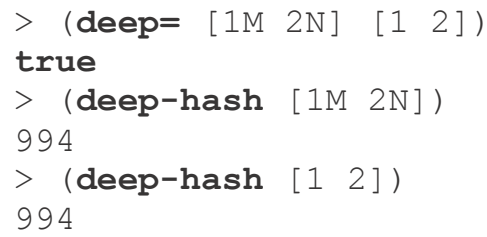

while with the default operators we have erroneous:

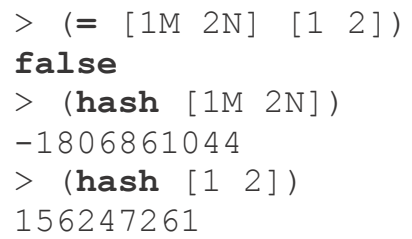

Unfortunately, with persistent sets and maps, things go wrong:

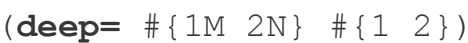

No implementation of method: :binary-deep= of protocol:

\#'kongra.identity/WithDeep= found for class: clojure.lang.PersistentHashset [Thrown class java.lang.IllegalArgumentException] ${ }^{2}$

and similarly:

(deep $\left.=\{1 \mathrm{M} 2 \mathrm{~N}\} \quad\left\{\begin{array}{ll}1 & 2\end{array}\right\}\right)$

No implementation of method: :binary-deep= of protocol:

\#'kongra.identity/WithDeep= found for class: clojure.lang.PersistentArrayMap [Thrown class java.lang.IllegalArgumentException] ${ }^{2}$

1 In an opposition to other sequences, vector elements may be randomly accessed in constant time using indexes, yet the vectors are not lazily evaluated, like other types of sequences may be.

2 Observable when being run either in a standalone application or in the Clojure REPL 
The same lack of implementation occurs for kongra.identity/deep-hash operator. As expected, the default Clojure equivalence and identity operators, exhibit wrongful behavior:

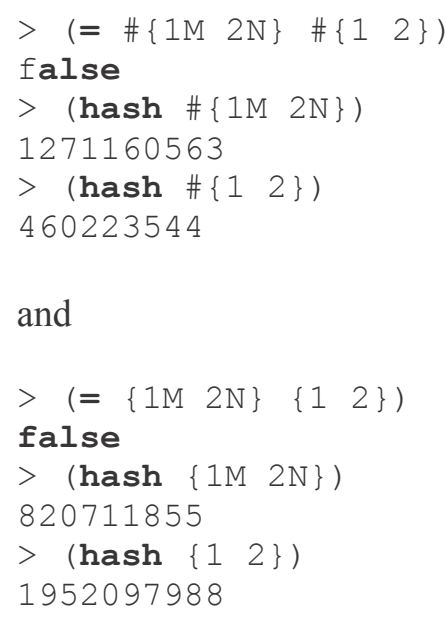

and

A thoughtful reader might ask was this lack of implementation an accident or a deliberate decision. The "deep" operators throw errors after all when called with arguments of unsupported kinds. In fact it was a deliberate decision dictated by the following two considerations:

1. There should be no default and "safe" implementation for objects of arbitrary types. Raising errors allows to find out and eliminate unpredictability in the system eagerly.

2. It is impossible to provide the implementation of the customizable equivalence and identity operators for arbitrary set or map type.

The second point is particularly interesting. In the case of sequences and lists (see [1]) the way to achieve the desired functionality was iterating over the elements of the collection and either aggregating their deep-hash values into a resulting deep-hash for the sequence or testing for the deep= (equivalence) of the (deeply) compared sequences. This approach must be extrapolated onto sets and maps as it seems to be the only reasonable implementation strategy; a deep-hash or deep = for any collection is a derivative of deep-hash or deep $=$ of it's components. Unfortunately, due to various ways a set or a map may be implemented (hash codes, balanced trees), there is no easy way 
to provide the "deep" semantics into these kinds of collections ${ }^{3}$; one can't inject neither deep-hash nor deep $=$ into their internal workings ${ }^{4}$.

All this led to making another undertaking of implementing special flavors of persistent sets and maps characterized by:

- possessing the deep-hash/deep= semantics,

- generosity in their implementation,

- ability to derive the actual container implementation by relying transparently on a specific back-end collection.

The following sections constitute a detailed description of the design decisions and a presentation of their implementation details.

\section{DeepEntry}

The major idea behind the realization of our sets and maps was to create wrapper instances that would be the actual representations of the objects to store within the container. Using this approach we could forget about reaching for the storage internals at the same time not loosing the ability to inject the customizable equivalence and identity semantics. We may call the wrapper instances the proxy objects (behind the proxy design pattern as described in [9]), because they allow to modify the original behavior of the stored objects.

The proxy type is called DeepEntry. The instances of this class are immutable POJO (Plain-Old Java Objects). The following listing shows the static structure of the class:

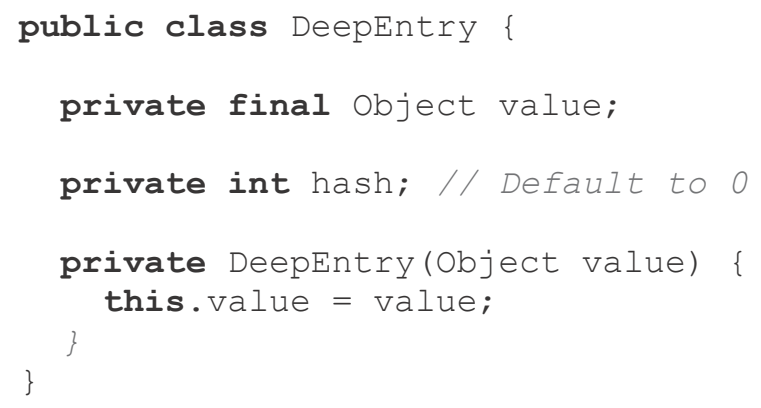

3 In an opposition to a simple sequence or list a set or map exhibits a whole variety of "views" of it's data in a form of iterators, entry sets, etc. It is a natural and justifiable expectation to these derivative "views" to also provide the "deep" semantics.

4 Testing a set or a map for containing a specified element is the most problematic functionality, as the test is associated with using deep-hash and/or deep= instead of default java.lang.Object methods hashCode and/or equals in the core storage for these data structures. 
Deep entries simply store the value and they offer caching of hash codes, like other immutable Java classes, e.g. java.lang.String. The two overridden methods are the core of the implementation:

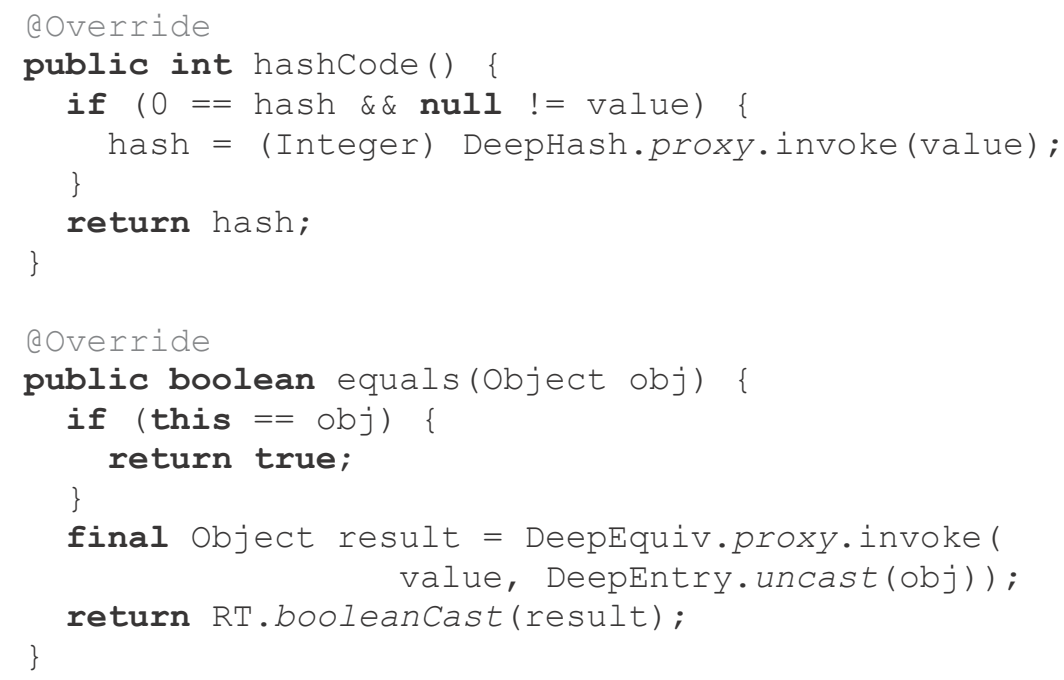

As it can be seen, both the identity as well as the equivalence operators expressed this way use the deep-hash and deep= procedures, represented here by special Java constants DeepEquiv.proxy and DeepHash.proxy.

DeepEntry belongs in fact to the implementation internals, it has a private constructor, but still there are the useful operators that allow any object to be coerced to a DeepEntry:

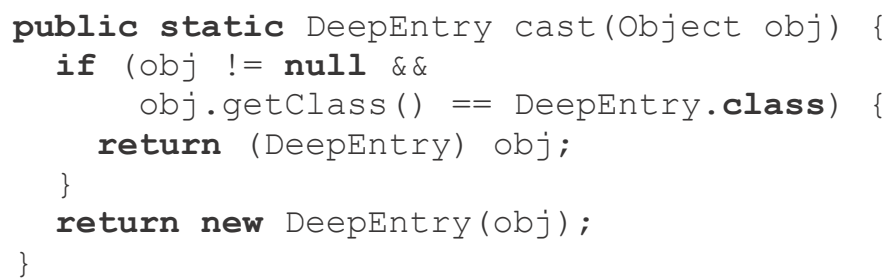

and uncasted afterward if needed:

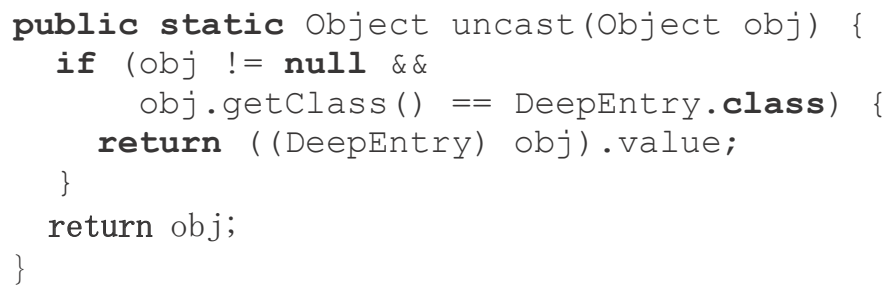


The latter mechanism is also extended to a form of a Clojure procedure, as this procedure is a part of the implementation (referenced to in the following sections):

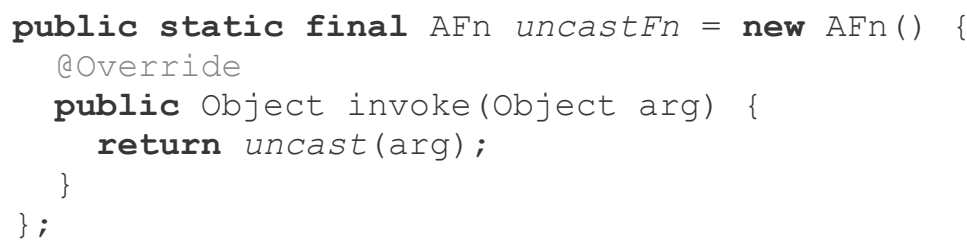

\section{DeepSet}

A DeepSet, the set that uses the customizable equivalence and identity semantics is the first kind of collection to be described. In fact it's internals are relatively simple to present.

The construction of the class mimics the implementations of other persistent sets within the standard Clojure library - the class is effectively nonextendable (by the presence of only private constructor), it has the value semantics and it implements a set of useful interfaces:

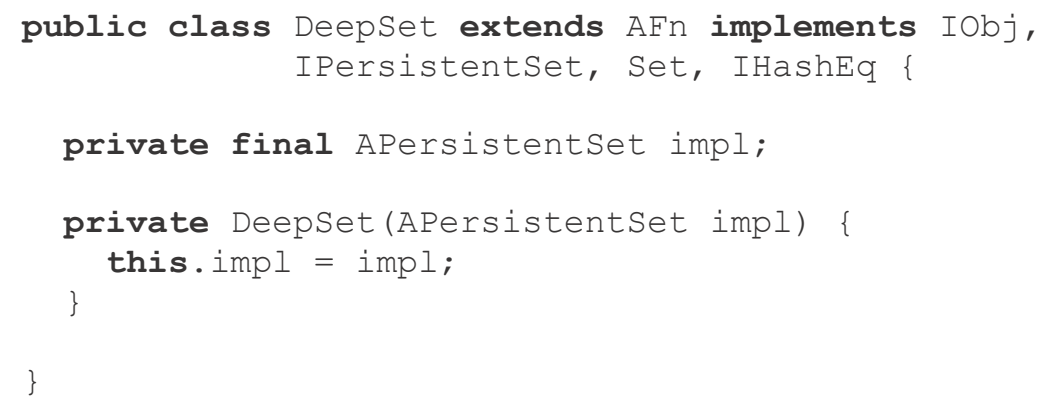

As it can be seen above, a DeepSet instance wraps a persistent set (the class clojure.lang.APersistentSet is the persistent set abstraction) called impl here. All objects stored in the DeepSet are being actually put into the persistent set after being coerced to a DeepEntry. This is a design pattern common both for DeepSets and for their associative counterpart - the DeepMap (see the next section). A manifestation of this approach can be seen at the following listing presenting the process of creating the set:

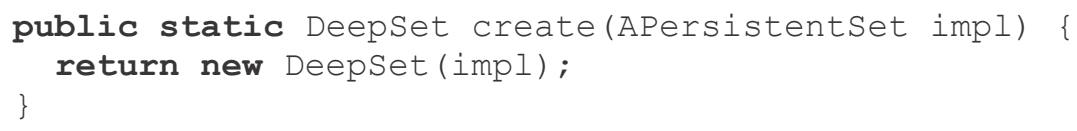




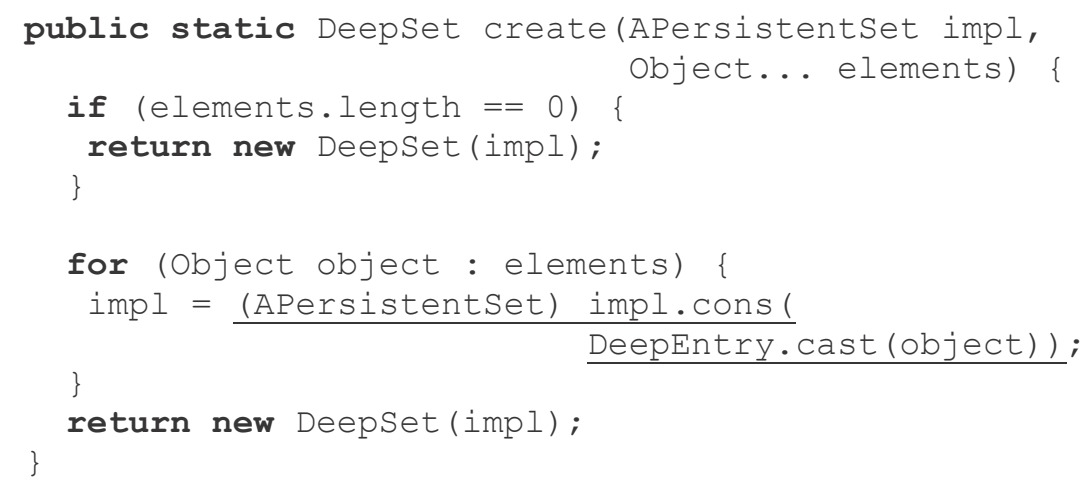

The underlined part of the code is the process of performing the actual storage of an object with simultaneous coercing to DeepEntry.

With the presence of this approach, implementing equivalence operators for DeepSet is trivial; it boils down to calling proper equivalence operators on the underlying impls.

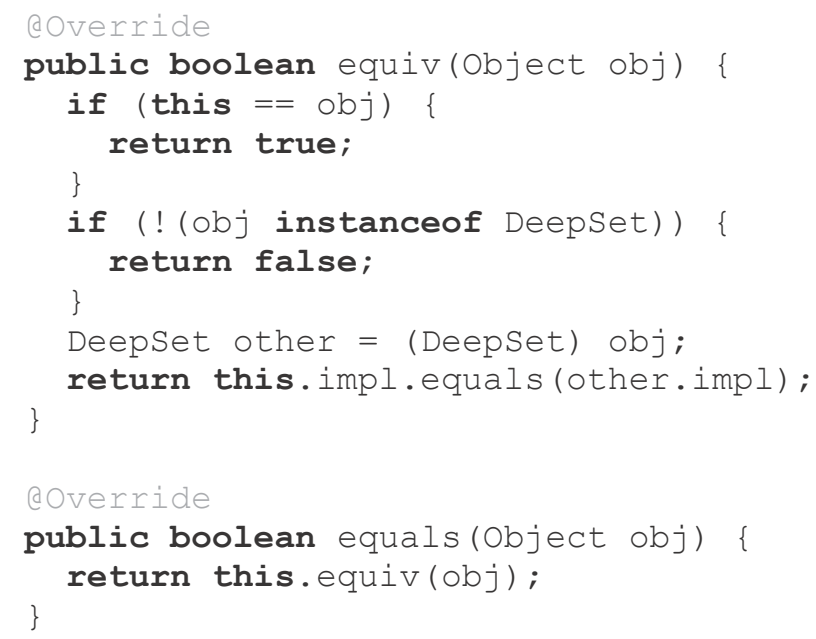

The same can be told about computing the hash code using the customizable mechanisms - the work is done in the $\mathbf{i m p l}$ with the presence of DeepEntries and their semantics:

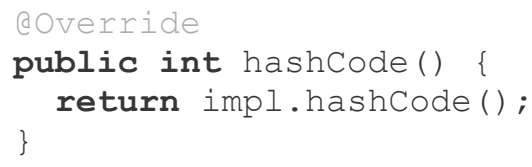




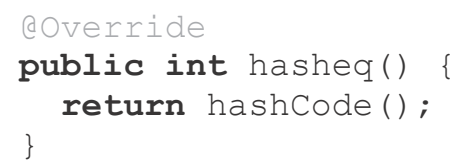

On the Clojure side there is a collection of mechanisms for creating DeepSet instances:

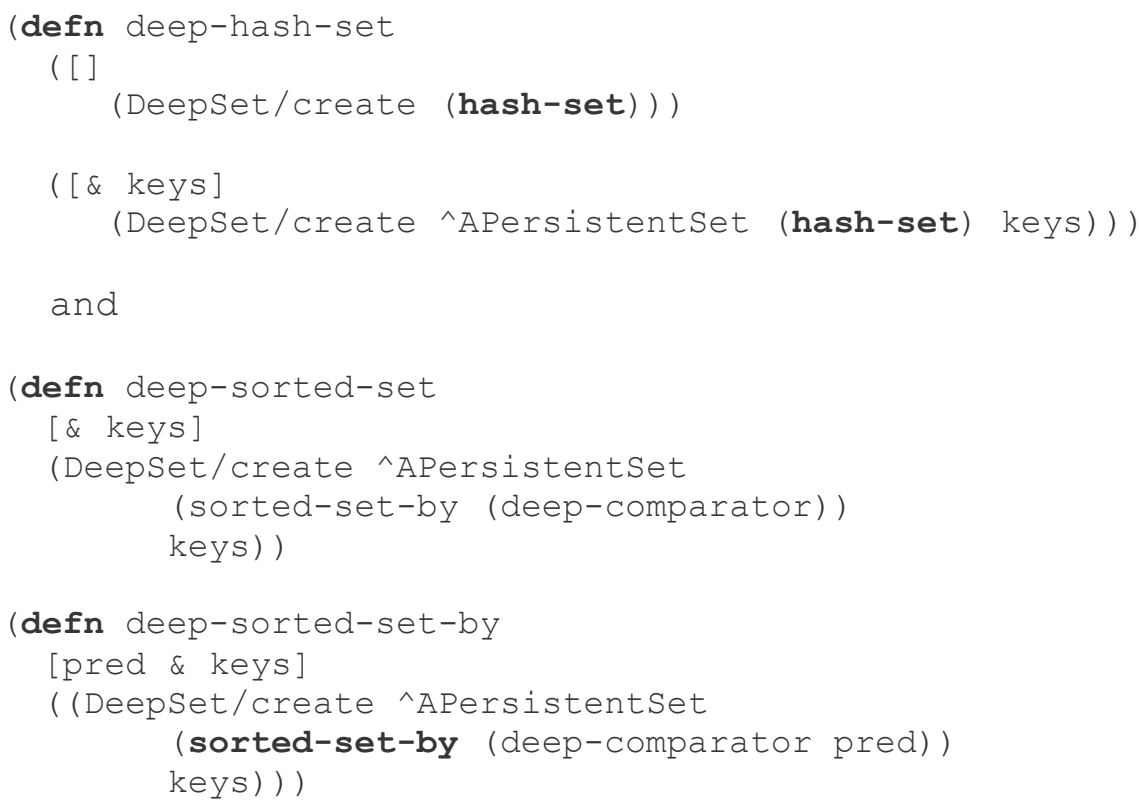

The latter two implemented with the help of a special constructor of DeepEntry aware comparators:

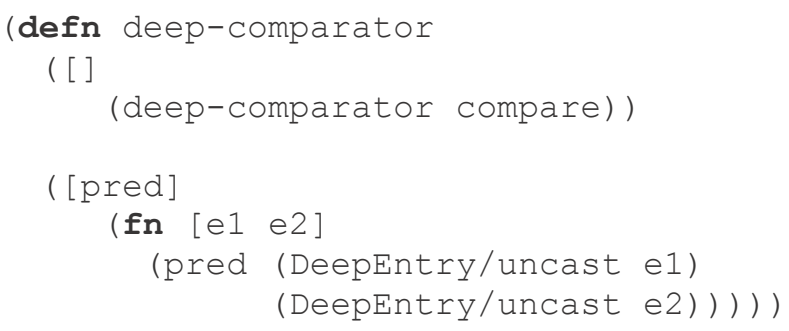

There are also the procedures for casting collections into DeepSets:

(defn deep-set?

[x]

(instance? Deepset $\mathrm{x}$ )) 


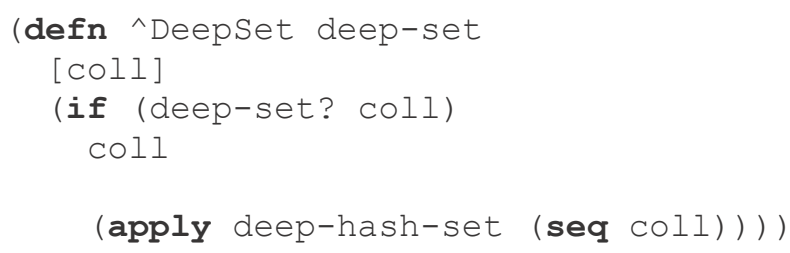

and a predefined empty DeepSet:

(def EMPTY-DEEP-SET (deep-hash-set))

All the destructive java.lang.Set operators are prohibited on DeepSet, as it is a persistent, immutable type. This is why:

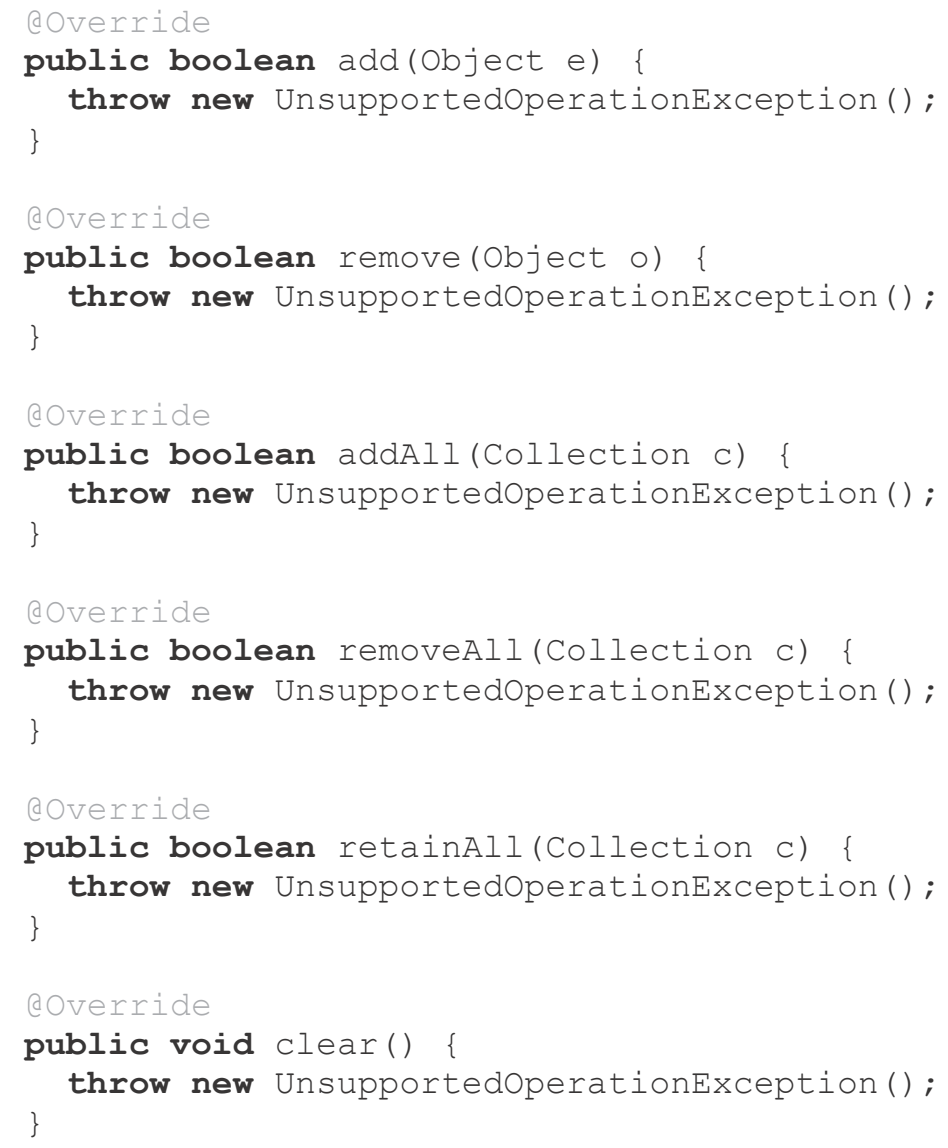


Finally, after extending the proper protocols ${ }^{5}$, namely WithDeepHash and WithDeep $=($ see [1] $)$, we get:

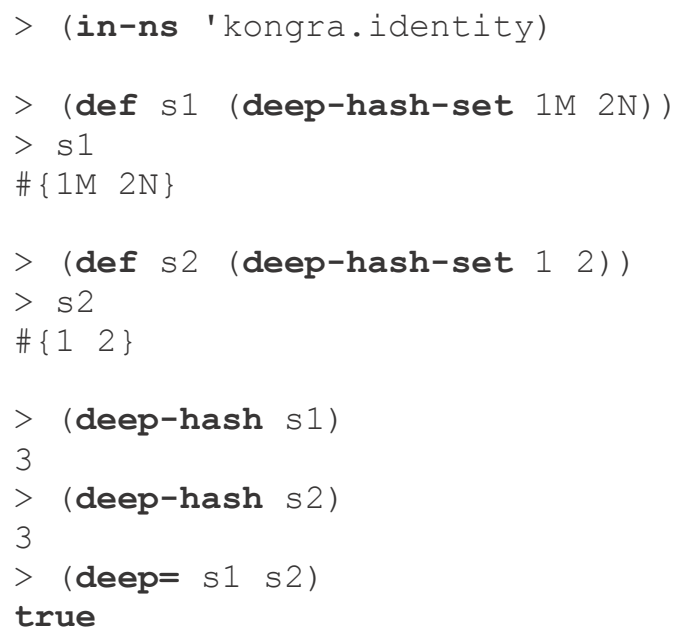

Additionally, the semantics expands onto the standard Clojure identity and equivalence operators:

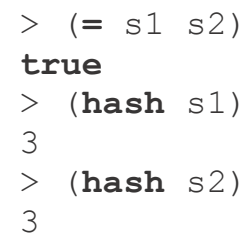

\section{DeepMap}

The implementation of an associative container differs from the DeepSet mostly in the fact that here we store both keys and values, both wrapped within the DeepEntry. The DeepMap also uses impl - an internal back-end storage, but here it is a persistent map (clojure.lang.APersistentMap derivative):

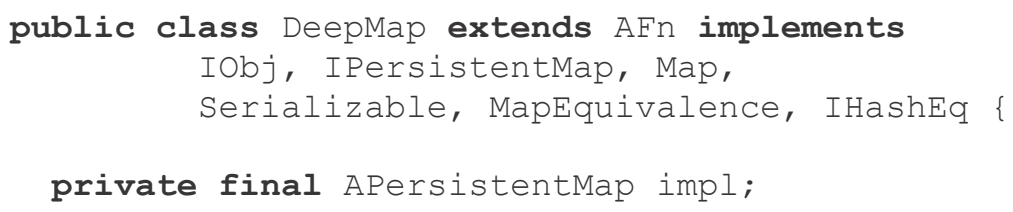

5 Tedious to present here due to a large amount of source code. For more, see the kongra/identity.clj compilation unit. 


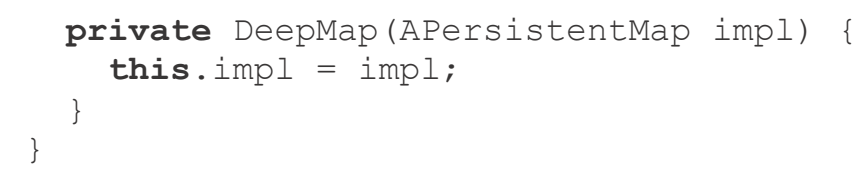

The identity (hash) and equivalence operators are trivial, as in the case of the DeepSet:

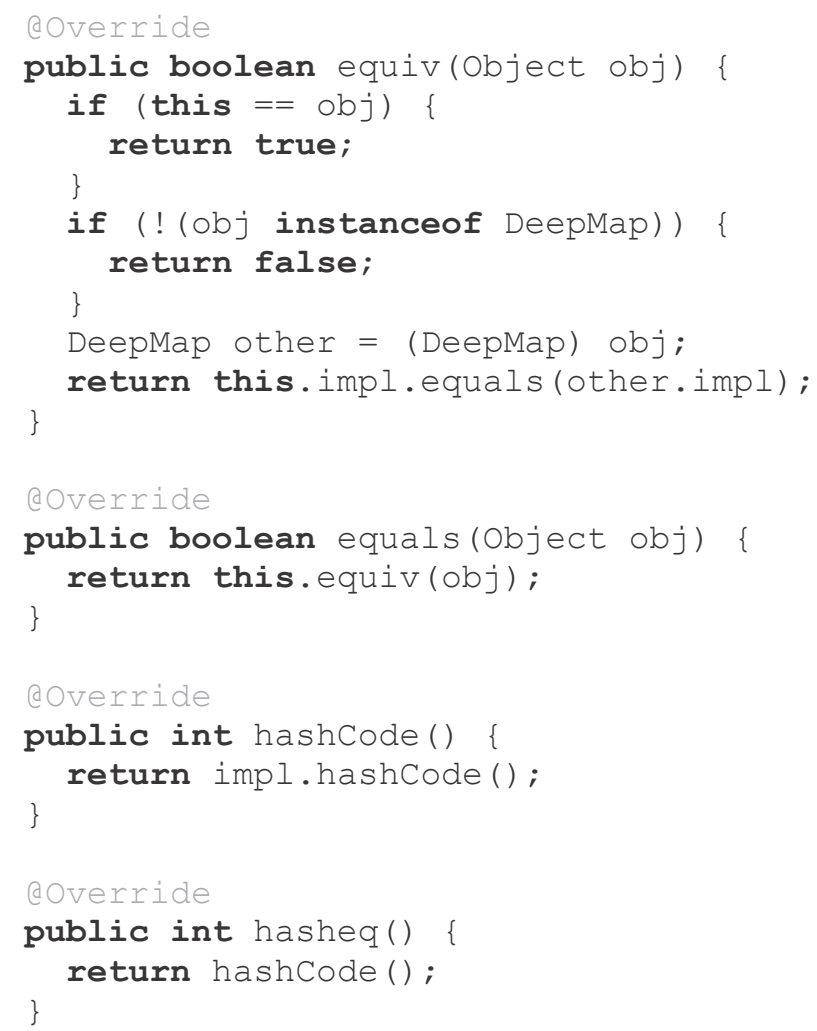

Creating DeepMaps is associated with performing the process of putting the keys and values into the $\boldsymbol{i m p l}$ after wrapping them. One can observe this at the following piece of code:

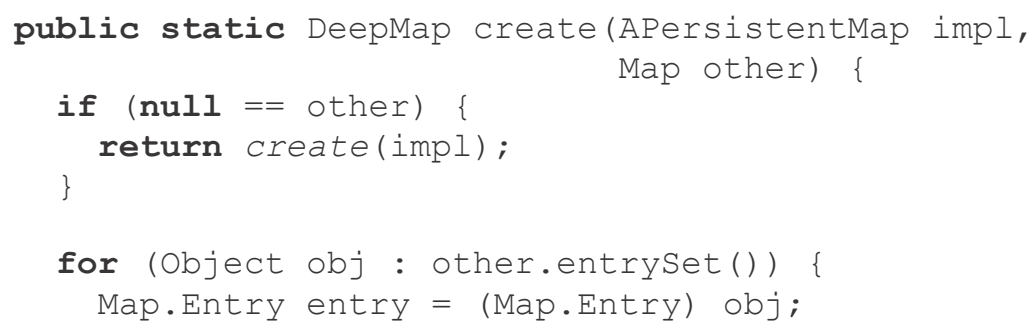


Grzanek K.

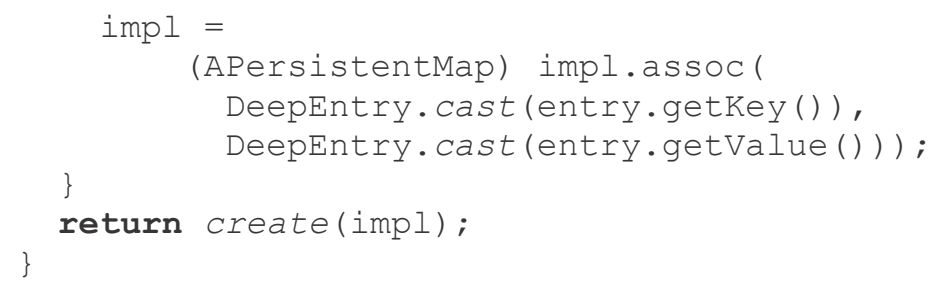

For the associative containers we also have a collection of operators on the Clojure side, that allow to create the "deep" maps:

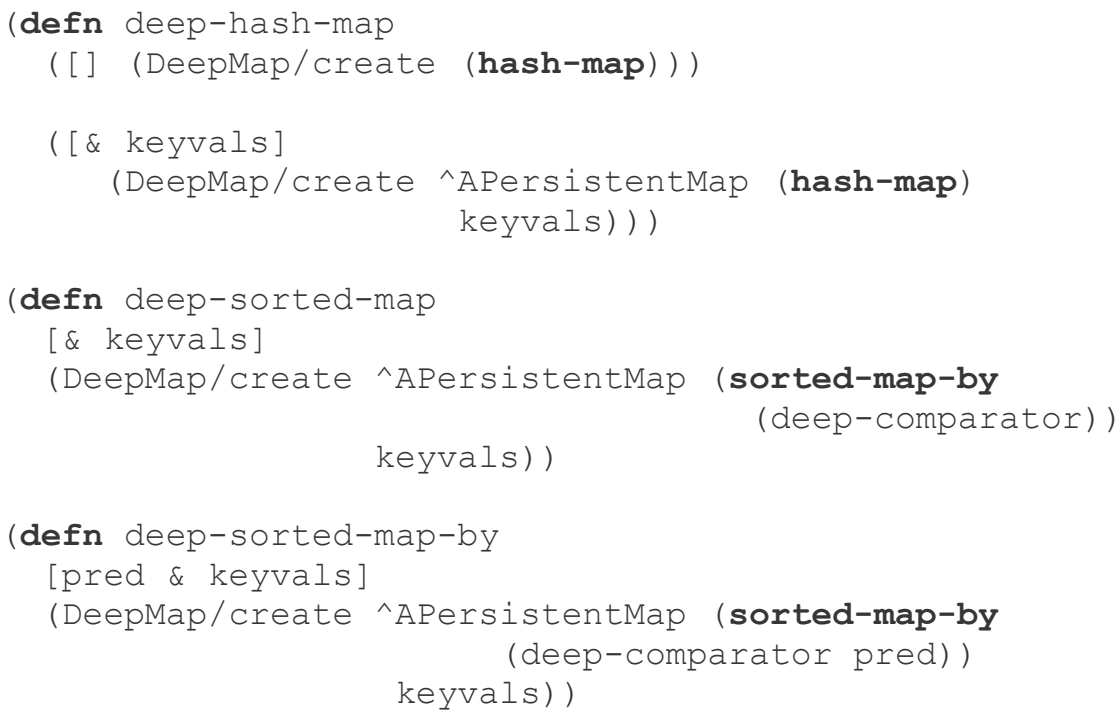

or casting arbitrary maps into their "deep" counterparts:

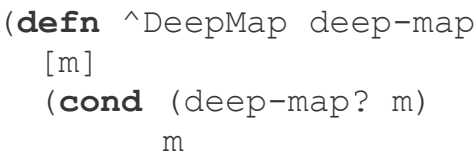


Persistent Collections With Customizable ...

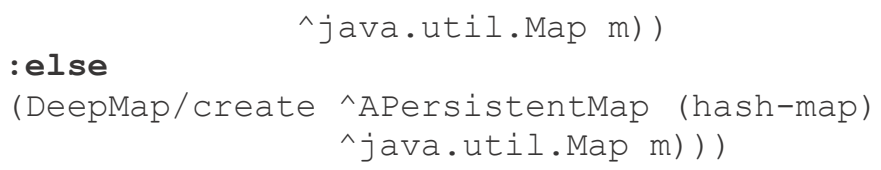

As in the case of the DeepSet, DeepMaps ensure their non-destructive nature by raising exceptions on an attempt to call destructive operators (belonging to the java.util.Map contract). For the sake of simplicity we do not present the related source codes here.

Testing for keys and values containment is as trivial as in the case of the identity and equivalence implementation after wrapping the arguments withind DeepEntry:

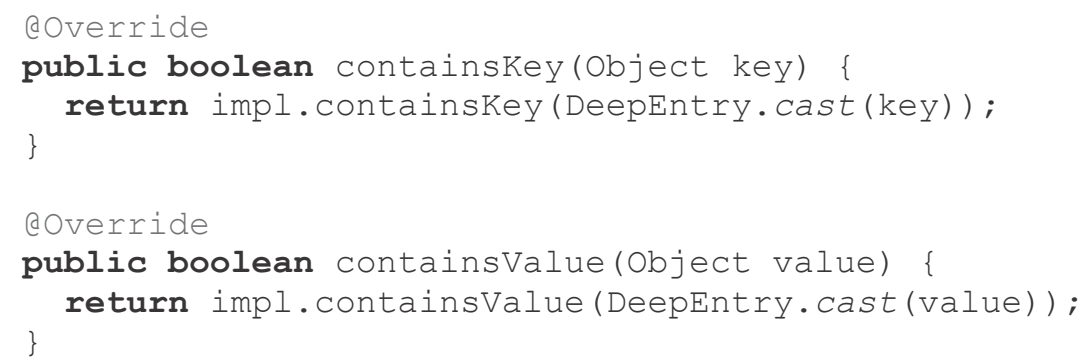

and similarly in the case of retrieving values stored under the given keys:

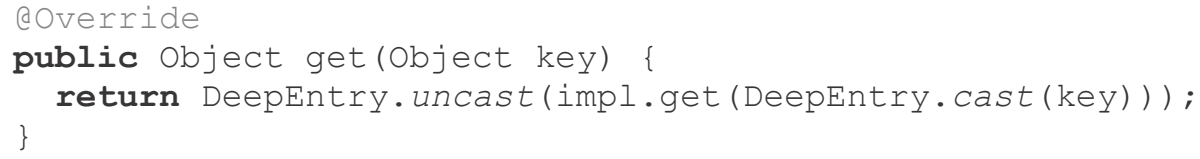

One final interesting implementation aspect is the algorithm for building the derivative collections of map entries, keys and values. They are all built around the same pattern - instances of kongra.utils.decorators.ImmutableDecoratingSet ${ }^{6}$ are used as shown below:

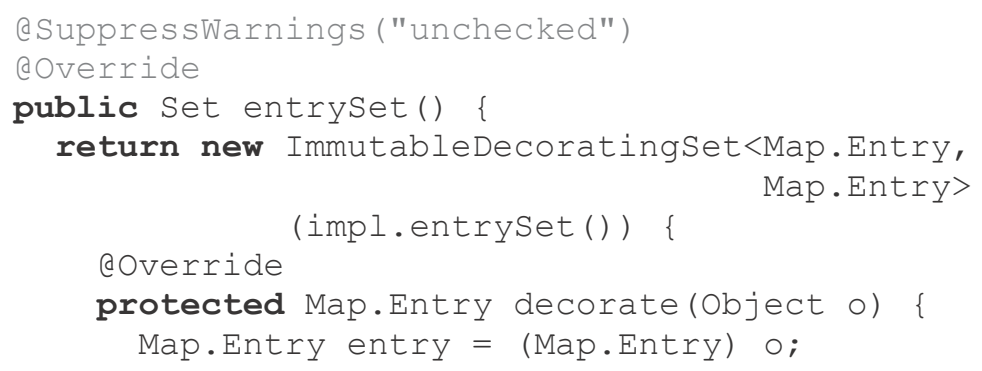

6 Full presentation of this interesting collection, whose range of possible applications is by no means limited to the functionality presented here, goes beyond the scope of this article. 
Grzanek K.

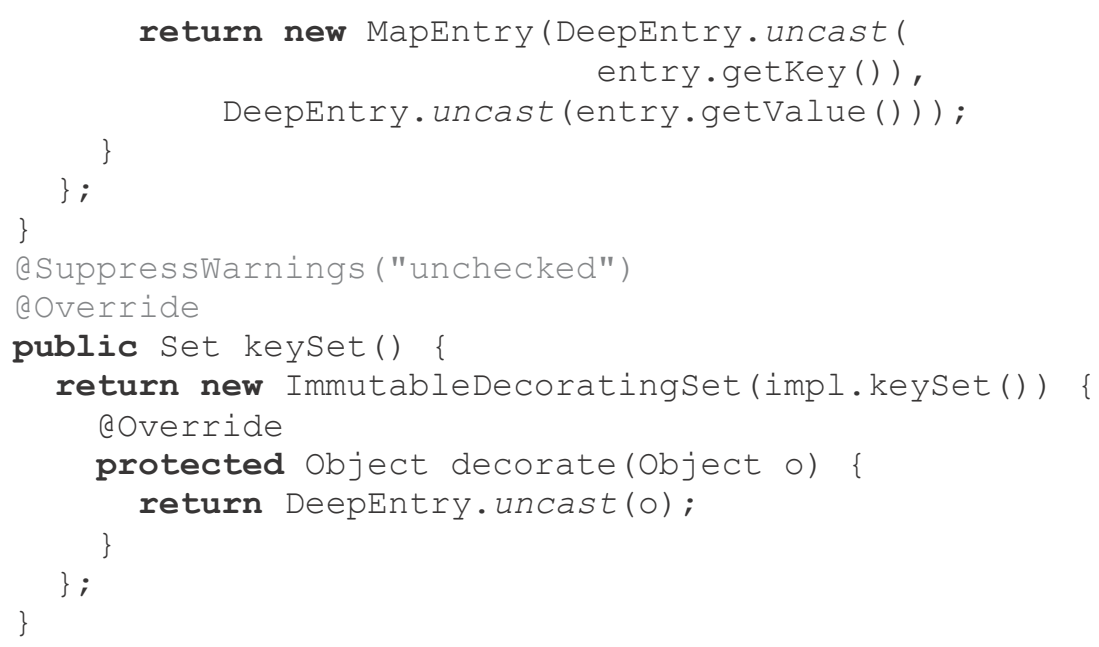

or - in the case of java.util.Map.values method implementation - the kongra.utils.decorators.ImmutableDecoratingCollection ${ }^{6}$ is used instead:

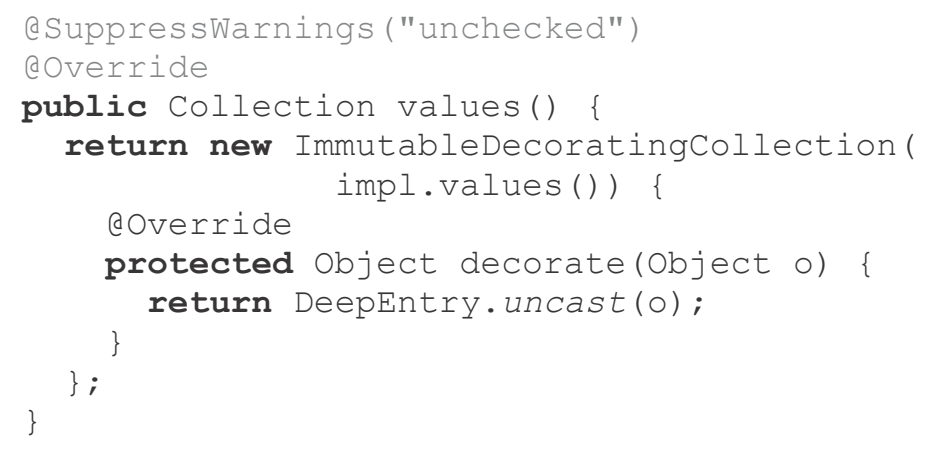

There are few more elements of the implementation of the DeepMap container whose presentation in this paper was postponed for the sake of the overall clarity.

Using the container is straightforward and it leads to the desired behaviors:

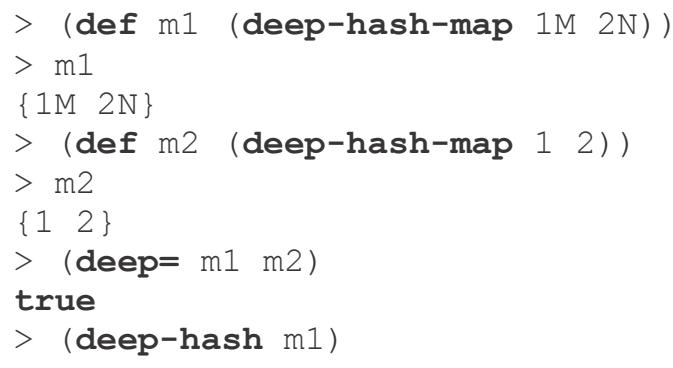




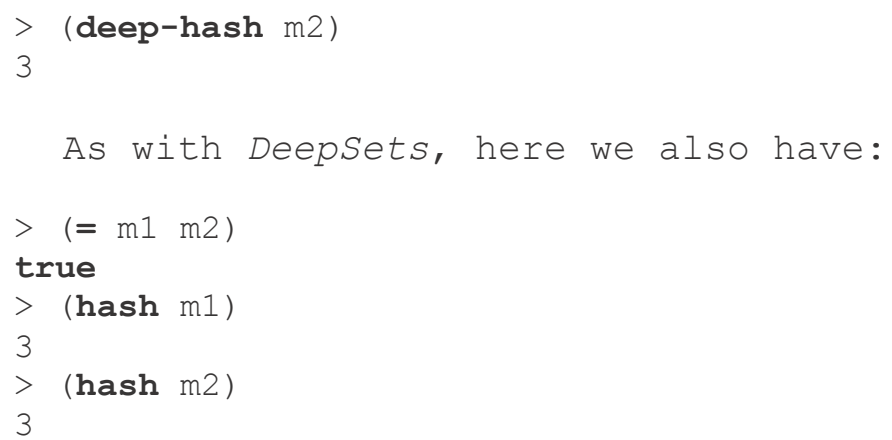

\section{Conclusions}

This paper finalizes a series of articles related to the customizable equivalence and identity in Clojure. The presented mechanisms should be used where needed, optionally extended on demand by modifying and/or extending the protocols presented in [1] and referenced to in this article. Establishing the specific performance profiles of the algorithms shown is left to the discretion of their users.

\section{References}

1. Grzanek K., 2013, Identity in Java and Clojure, Design and Implementation Considerations, Journal of Applied Computer Science Methods, No. 2 Vol. 5 2013

2. Oracle, 2014, Java ${ }^{\mathrm{TM}}$ Platform, Standard Edition 8 API Specification, http://docs.oracle.com/javase/8/docs/api/

3. Gosling J., Joy B., Steele G., Bracha G., 2005, The Java ${ }^{\mathrm{TM}}$ Language Specification Third Edition, ISBN 0-321-24678-0, available at the Oracle Technology Network (2014) http://docs.oracle.com/javase/specs/

4. Halloway S., 2009, Programming Clojure, ISBN: 978-1-93435-633-3, The Pragmatic Bookshelf

5. Emerick Ch., Carper B., Grand Ch., 2012, Clojure Programming, O'Reilly Media Inc., ISBN: 978-1-449-39470-7

6. Bagwell P., 2000, Ideal Hash Trees (Report), Infoscience Department, École Polytechnique Fédérale de Lausanne

7. Touretzky D.S., 1990, COMMON LISP: A Gentle Introduction to Symbolic Computation, The Benjamin/Cummings Publishing Company,Inc., ISBN: 08053-0492-4

8. Graham P., 1993, On Lisp - Advanced Techniques for Common Lisp, Prentice Hall; 1st edition (September 9, 1993), ISBN-10: 0130305529, ISBN-13: 9780130305527

9. Gamma, et al., E., 1995., Design Patterns. Reading, MA: Addison-Wesley Publishing Co, Inc. pp. 175ff. ISBN: 0-201-63361-2 